\title{
Pupil dilation as indicative of cognitive workload while driving a car: effects of cell phone use and driver experience in young adults
}

\author{
BETHÂNYA G. CARIZIO 1 | GUSTAVO A. SILVA | GABRIEL P. PASCHOALINO ${ }^{1}$ | JULIANA C. ANGELO² | GISELE C. \\ GOTARDI ${ }^{1}$ | PAULA F. P. ZAGO1 | SÉRGIO T. RODRIGUES1
}

${ }^{1}$ LIVIA- Laboratory of Information, Vision and Action, Department of Physical Education, Faculty of Sciences, São Paulo State University, Bauru, SP, Brazil.

2 LAPE- Exercise Prescription and Assessment Laboratory. Department of Physical Education, Faculty of Sciences, São Paulo State University, Bauru, SP, Brazil.

Correspondence to: Sérgio Tosi Rodrigues. Laboratory of Information, Vision and Action, Department of Physical Education, Faculty of Sciences, São Paulo State University, Bauru, São Paulo, Brazil.

email: sergio.tosi@unesp.br

https://doi.org/10.20338/bjmb.v15i5.269

\author{
HIGHLIGHTS \\ - Cognitive overload resulting from the \\ involvement of drivers in conversational \\ activities while driving affects the availability of \\ attentional resources, leading to poor \\ performance. \\ - Novice drivers had greater pupil dilation and, \\ consequently, greater cognitive overload than \\ experienced drivers when performing the \\ conversation task while driving a car. \\ - Experienced drivers had a longer time to \\ reach peak pupil dilation compared to novices \\ when performing the conversation task while \\ driving a car, indicating less cognitive overload.

$\begin{array}{ll}\text { ABBREVIATIONS } \\ \text { ASL } & \begin{array}{l}\text { Applied Science Laboratory } \\ \text { driving without talking on the cell } \\ \text { phone, with both hands on the } \\ \text { wheel - control condition }\end{array} \\ \text { EXP } & \begin{array}{l}\text { Experienced group } \\ \text { FRE }\end{array} \\ & \begin{array}{l}\text { Driving and talking on a cell phone } \\ \text { in hands-free mode, with both } \\ \text { hands on the steering wheel } \\ \text { DAN }\end{array} \\ & \begin{array}{l}\text { Driving and talking on a cell phone } \\ \text { holding the device with right hand, } \\ \text { keeping the left hand on the } \\ \text { steering wheedriving without } \\ \text { talking on the cell }\end{array} \\ \text { NOV } & \text { Novice group } \\ \text { SPSS } & \text { Statistical Package for Social } \\ & \text { Sciences }\end{array}$

PUBLICATION DATA

Received 15112021

Accepted 30112021

Published 01122021
BACKGROUND: Cognitive workload resulting from drivers' engagement in concomitant tasks while driving, such as talking on a cell phone, affects the availability of attentional resources for the various stages of information processing, which can interfere with the selection of relevant traffic information, leading to poor performance and higher risk of accidents.

AIM: The purpose of this study was to test the adaptation and application of the method of fixation-aligned pupillary response averaging to the car driving context, and, if successful, to determine effects of talking on a cell phone while driving, in both handheld and hands-free situations, and effects of driving experience on pupillary responses of young adult drivers, as indicative of cognitive workload.

METHOD: Ten novice and ten experienced drivers had pupil diameter measured while driving in a car simulator under velocity of $80-120 \mathrm{~km} / \mathrm{h}$, daylight, linear trajectory and low traffic level. Data analysis was based on the method of fixation-aligned pupillary response averaging.

RESULTS: Noise curves were around baseline (zero) values while pupil dilation curves clearly stood out from noise magnitude, in all conditions for both groups. Greater pupil dilation peak during talking on the cell phone (handheld and hands-free conditions) while driving occurred only for the novice group.

CONCLUSION: Adaptation and application of the method of fixation-aligned pupillary response averaging to the car driving context succeed. Cognitive workload imposed by the dual task of talking on a cell phone increased pupil dilation for novice drivers, which may alter acquisition of visual information and impair driving behavior.

KEYWORDS: Cognitive workload | Driving simulator | Pupillometry | Dual task | Cell phone

\section{INTRODUCTION}

Pupil diameter increases with increasing mental effort and systematically reflects the burden of processing tasks and allocation of resources in various domains of cognitive

\begin{tabular}{l|l|l|l|ll}
\hline Carizio et al. & 2021 & VOL.15 & N.5 & https://doi.org/10.20338/bjmb.v15i5.269
\end{tabular}




\section{5 years of Brazilian Journal of Motor Behavior}

functioning. ${ }^{1}$ An individual experiencing an increase in cognitive workload (e.g., surveillance or short-term memory) has a resulting brain activity which dilates the pupils via the sympathetic system. ${ }^{2}$ Increase in cognitive workload due to tasks such as talking on a cell phone has been observed in the literature, confirming that it interferes with the attention mechanism and tends to disrupt drivers' perceptual and motor behavior, increasing risk of accidents. ${ }^{3}$ On the other hand, experience and automaticity in driving tends to improve the driver's visual search strategy according to the complexity of the road and context, favoring the perception of imminent dangers, and modulating the driver's responses to increased cognitive workload. ${ }^{4}$ In short, measures of pupil dilation seem capable of revealing interactions among cognitive effort, ongoing task demands, and experience level. Deleterious effects of increased cognitive workload on perceptual-motor performance are well-known; however, how they can be attenuated by experience during driving is still a matter of debate. 4,5

Methods of classifying and predicting cognitive workload during driving have explored linkages between gaze characteristics and pupil diameter data. ${ }^{6}$ For instance, Klingner $\mathrm{r}^{7,8}$ proposed a novel analysis of pupil measurements made in conjunction with eye tracking, which was termed fixation-aligned pupillary response averaging. This technique allows measurement of quick changes in cognitive workload during visual tasks, when task components occur at unpredictable moments but are identifiable via gaze data; thus, short continuous pupil data epochs can be selected based on fixations on a particular spot or a scan path, temporally aligned, and averaged together. The alignment procedure is made by temporal translation and linear warping; these two aspects are used to place corresponding parts of the gaze patterns at corresponding times and then average them together. Klingner illustrated his proposal by analyzing the tasks of visual search and map reading; the present study adapted his work, applying it to the car driving task.

Traffic safety is directly related to the mental effort required from the driver. Performing secondary tasks such as using cell phone while driving is an additional activity that dissipates attention and adds to the drivers' workload. This study offers novel method to analyze pupil responses during driving and tests effects of dual-task and experience in this context. Particularly, the purpose of this study was twofold: (i) to test the adaptation and application of Klingner's method of fixation-aligned pupillary response averaging to the car driving context, and, if successful, (ii) to determine effects of talking on a cell phone while driving, in both handheld and hands-free situations, and effects of driving experience on drivers' pupillary responses, as indicative of cognitive workload. First, as indicative of successful method application, we expected that pupillary response average curves would clearly, through visual inspection, stand out in comparison to a noise measurement, the plus/minus average used by Klingner. Then, we hypothesized that talking on a cell phone while driving, in both handheld and hands-free situations, would significantly increase pupillary responses, mainly in the novice group.

\section{METHOD}

\section{Participants}

Ten novice drivers and ten experienced drivers, all right-handed, with normal or 


\section{5 years of Brazilian Journal of Motor Behavior}

corrected to normal vision, with no history of muscular, neurological, postural, and locomotor deficits, participated in the study. All volunteers signed a free and informed consent form approved by the local ethics committee. The novice group (NOV) consisted of five men and five women, with mean age of $21.0(S D=3.1)$ years, and mean driving experience of 2.8 $(S D=2.7)$ years; the experienced group $(E X P)$ consisted of five men and five women, with a mean age of $31.1(S D=12.0$ ) years, and mean driving experience of $11.1(S D=6.0)$ years. Estimation of driving experience was based on method used by Lehtonen and colleagues ${ }^{9}$ and Summala and colleagues 10 ; calculation of mileage traveled was derived from questionnaire regarding daily driving behavior provided by participants. NOV group was composed by participants who drove up to $15,000 \mathrm{~km}$ while EXP participants drove above $30,000 \mathrm{~km} \cdot 9,10$

\section{Equipment}

Drivers had their eye kinematics and pupil diameter synchronously recorded with an eye tracker system (Applied Science Laboratory - ASL, model H6), while driving in the driving simulator system (City Car Driving, Forward Developments, version 1.0), with images projected to a flat-screen 46-inch television, under conditions of $80-120 \mathrm{~km} / \mathrm{h}$ speed, daylight, linear trajectory and low traffic level, connected to a cockpit (Logitech) containing a seat, sixspeed gearbox, steering, accelerator, brake, and clutch pedals, as illustrated in Figure 1. Software ASL Results Plus (version 1.8.2.18, Applied Science Laboratory - ASL), Matlab (Mathworks, version 7.10.0.499), and SPSS (Statistical Package for Social Sciences, version 17.0.1) were, respectively, used for fixation analysis, all calculations, and statistical analysis.

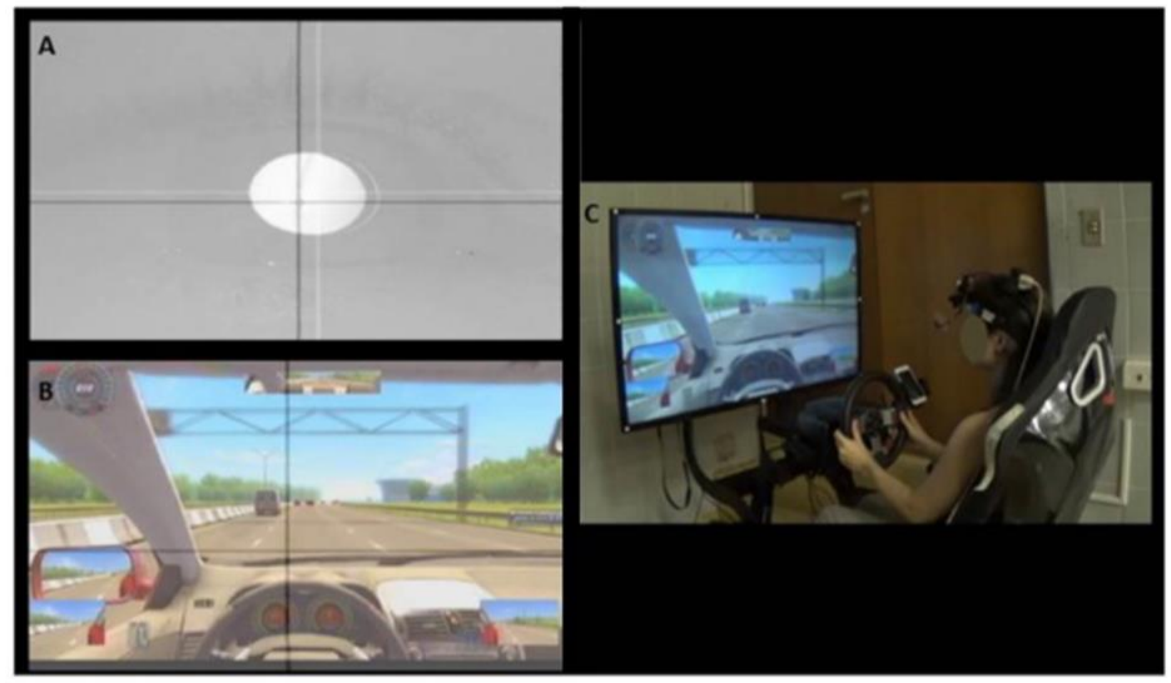

Figure 1. Illustration of sources of video available during data collection: $(A)$ image of the eye and horizontal and vertical coordinates of the centroid of the pupil and corneal reflection, $(B)$ image of the respective coordinates of the line-of-gaze on the simulator scene, and $(C)$ external camera image showing a participant and experimental setup.

\section{Procedures}

Prior to data collection, participants went through a familiarization procedure 
involving experience with all pieces of equipment (cockpit and simulator systems, cell phone, and eye tracker components), their respective adjustment, and general instructions, which was followed by gaze calibration procedures and a four-minute pre-driving practice.

Task. Participants were subjected to three experimental conditions: driving without talking on the cell phone, with both hands on the wheel - control condition (CON), driving and talking on a cell phone holding the device with right hand, keeping the left hand on the steering wheel (HAN), and driving and talking on a cell phone in hands-free mode, with both hands on the steering wheel (FRE). The order of conditions was randomized to avoid possible effects of order. In all conditions, participants were instructed to drive naturally, following the national traffic laws. In both cell phone conditions (HAN and FRE), participants had to answer the phone and maintain a live conversation with a researcher, answering simple questions, with contents such as the name of the participant, their parents or relatives, and what they like to do in their leisure time. Different conversation scripts were created and randomized across conditions and groups. One single trial of approximately four minutes was recorded for each participant in each condition.

\section{Data analysis}

Eye tracking raw data $(60 \mathrm{~Hz})$ provided pupil diameter combined with onset and offset of each single fixation throughout the trial. Each fixation was defined by determining its onset and offset. Via ASL Results Plus software, fixation onset was defined as twice the standard deviation of gaze position (95\% confidence interval) less than one degree of visual angle (horizontal and vertical) for $100 \mathrm{~ms}$ (seven data points); fixation offset was defined as three data points off from the initial fixation value by more than one degree of visual angle (horizontal and vertical). A specific routine was written in Matlab environment to apply Klingner's method of fixation-aligned pupillary response averaging.7,8 Each trial consisted of 60 seconds of data during the car driving task (which was spatially located in the same approximate zone within the simulated trajectory); each participant performed only one trial per condition. A variable number of fixations were identified in each trial. Curves of pupil diameter data were selected with respect to fixation onset: each epoch started $500 \mathrm{~ms}$ prior to and ended $500 \mathrm{~ms}$ after fixation onset; these curves enabled the calculation of an average curve of the trial (and respective standard error values at each data point). The number of curves in each condition was equivalent to the number of fixations observed in that condition. Pupil dilation / constriction was determined with respect to a baseline established by calculating the average over the period of $500 \mathrm{~ms}$ prior to fixation onset; this average value was subtracted from all values of the respective curve. Although light conditions were controlled during data collection, the baseline procedure was used to determine the changes in pupil diameter with respect to the observed average pupil diameter prior to each fixation onset. The amount of noise involved in the averaging procedure was estimated through the calculation of a plus / minus average using the same curves mentioned above; the purpose of averaging aligned pupil dilation data is to preserve the signal of the task-evoked pupillary response while decreasing the power of signal components not correlated in time with gaze events (the noise). Usually, the magnitude of the pupillary response being investigated is not known a priori, which makes difficult to determine whether a feature of the averaged signal would be a noise or not. The solution for this type of problem (the plus / minus average) emerged from the analysis of averaged electroencephalogram data, which 
estimates magnitude of noise by itself; 11 this was originally described as plus / minus reference. ${ }^{12}$ According to Klingner, 7,8 instead of simply adding all the epochs and dividing by the number of epochs, the epochs are alternately added and subtracted from the running total (which is only defined for even number of epochs; in the present study, when the number of fixations in a given trial was odd, the last epoch was excluded from calculation). The plus/ minus average "is usually a good estimate of the noise power in the standard average. If no pupillary response stands out above this level, then either there is no pupillary response to see, or more trials are required to drive the noise power even lower." (p. 278). ${ }^{7}$

In order to illustrate the data reduction process, Figure 2 shows the average curve ( \pm standard error) of the CON condition of a participant in the EXP group, highlighting the peak of pupil dilation, composed, on the vertical axis, by the coordinate of the peak magnitude (variable "peak", filled circle) and, on the horizontal axis, by the coordinate of the time of occurrence of this peak (variable "tpeak", empty circle). Thus, values of peak and tpeak were obtained from each participant from both groups in each experimental condition. Data of each dependent variable were submitted to a Group (EXP, NOV) by Condition (CON, HAN, FRE) analysis of variance, with repeated measures in the second factor. Tukey Honestly Significant Difference tests, Greenhouse-Geisser degrees of freedom adjustments, and Bonferroni multiple-comparison probability adjustments were conducted when necessary. Alpha value was 0.05 .

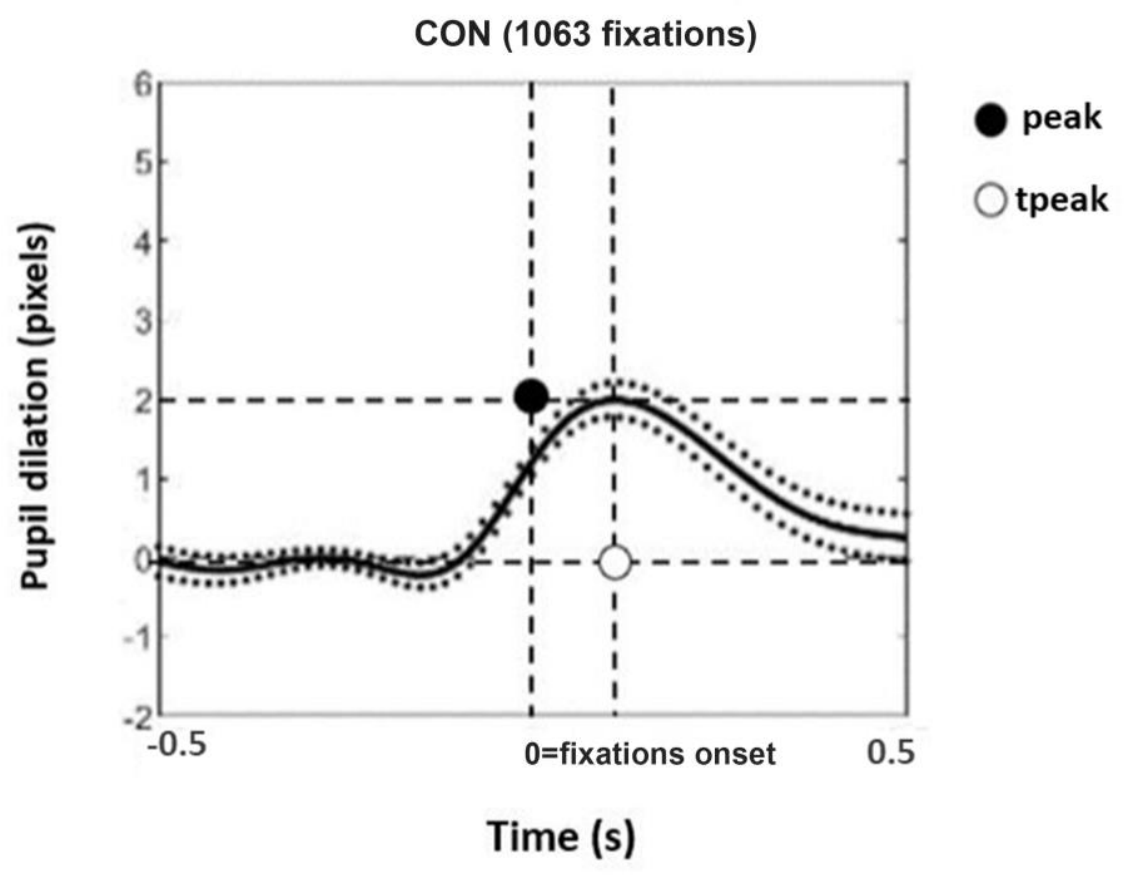

Figure 2. Illustrative plot of average curve ( \pm standard error) of pupil dilation (pixels) of an experienced driver during the control condition (CON) as a function of time (s), identifying the variables pupil dilation peak (peak) and time to pupil dilation peak (tpeak). Zero time equals fixation onset, characteristic used to temporally align 1063 curves represented. 


\section{RESULTS}

Figure 3 summarizes pupil dilation mean curves of both groups in each condition and their respective noise measurements. While noise curves are around baseline (zero) values, pupil dilation curves present a clearly distinct pattern above noise magnitude, in all conditions for both groups. The observable difference between pupil dilation and noise curves is indicative that the pupillary response signal was adequately captured via Klingner's method of fixation-aligned pupillary response averaging.
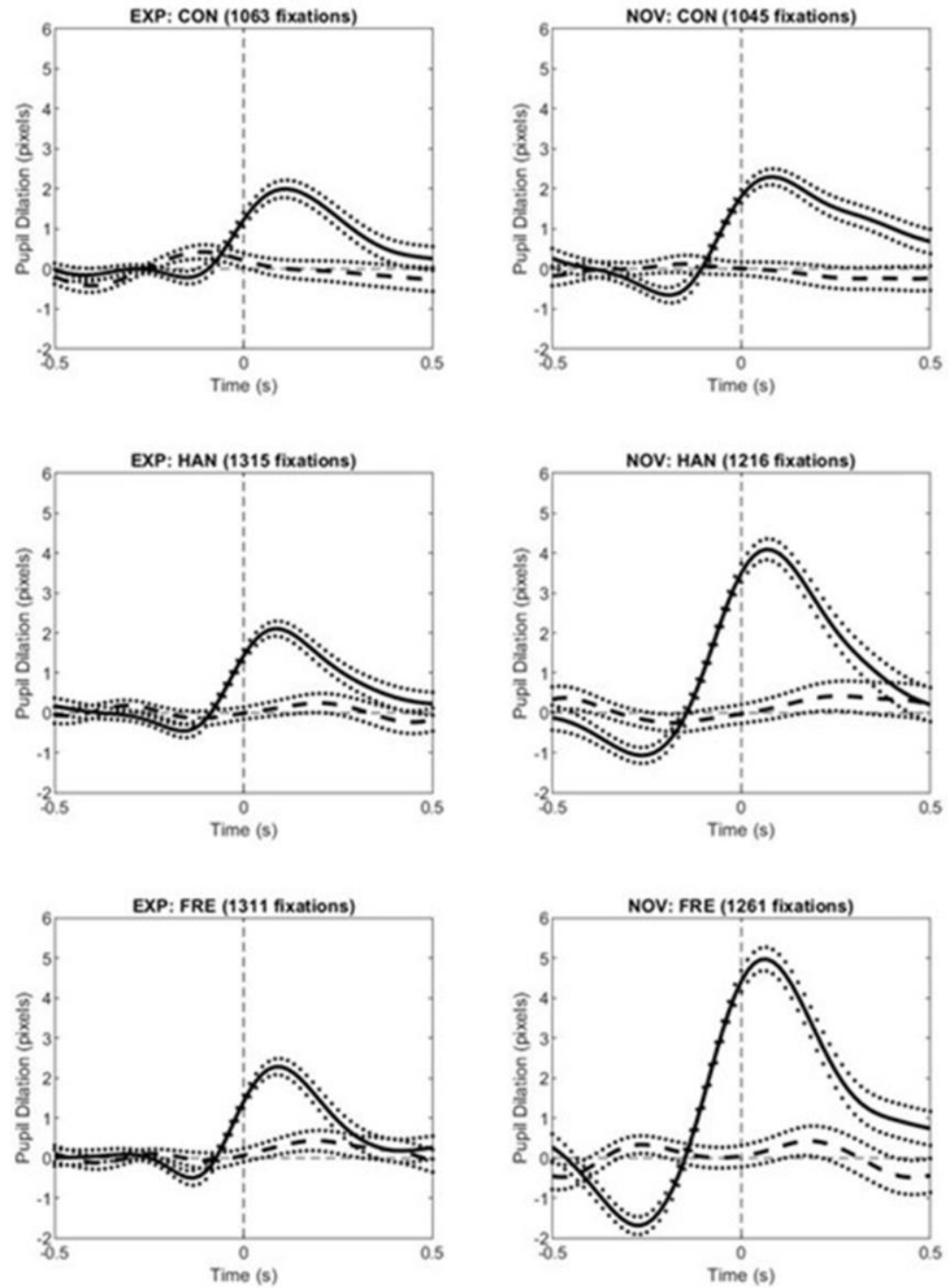

Figure 3. Pupil dilation (pixels) mean (solid line) and noise (dashed line) [ \pm standard error (dots)] of experienced (EXP) and novice (NOV) groups during control (CON), handheld (HAN), and hands-free (FRE) conditions. Zero time equals fixation onset, characteristic used to temporally align all curves represented (equal 
to number of fixations on the top of each plot).

Peak was significantly affected by the condition, $F(1.8,31.6)=3.64, p=0.043$. The post-hoc analysis revealed that no comparison between the pairs of conditions reached significance. Additionally, peak was significantly affected by the group factor, $F(1,18)=$ $6.91, p=0.017$, with EXP group showing lower peak $(M=2.46, S E=0.45)$ than NOV group $(M=4.13, S E=0.45)$. Peak was also significantly affected by the group-by-condition interaction, $F(1.8,31.6)=3.80, p=0.038$. The post-hoc analysis of the group-by-condition interaction revealed, only among NOV participants, a significant increase in peak in the HAN condition $(M=4.74, S E=0.58)$, and in the FRE condition $(M=5.16, S E=0.69)$, as compared to the CON condition $(M=2.50, S E=0.58), p=0.006$ and 0.007 , respectively, as shown in Figure 4.

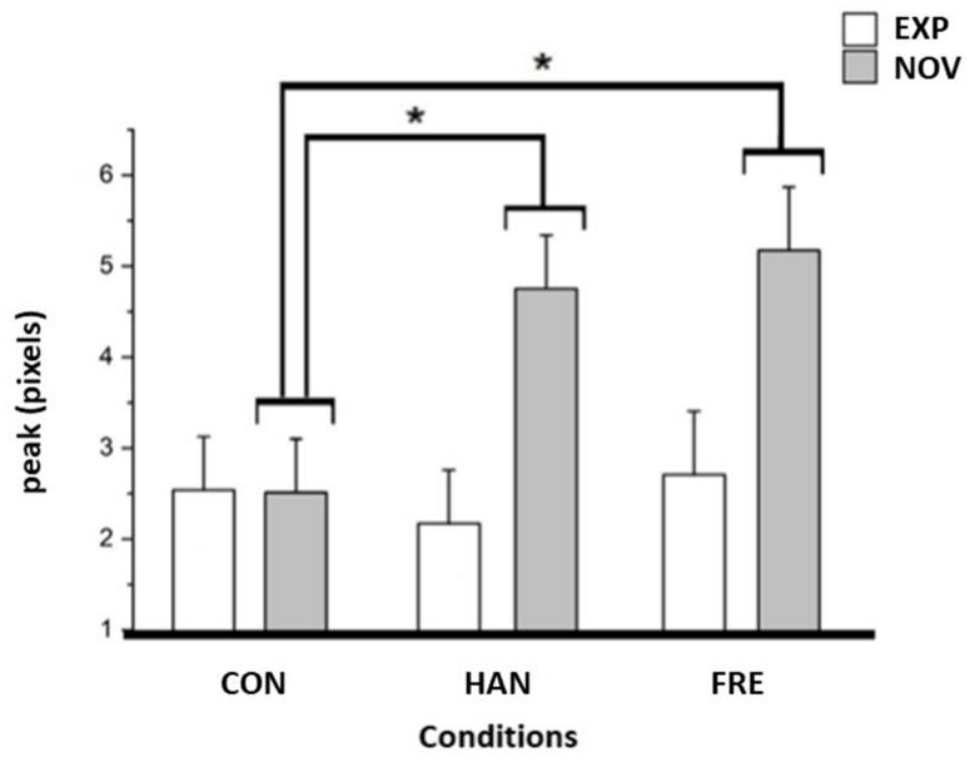

Figure 4. Mean (standard error) of peak (pixels) during control (CON), handheld (HAN), and hands-free (FRE) conditions of experienced (EXP) and novice (NOV) groups. ${ }^{*} p<0.05$.

Tpeak was significantly affected by the condition, $F(1.4,24.6)=7,00, p=0.008$. The post-hoc analysis revealed that tpeak was significantly higher in the CON condition ( $M$ $=0.109, S E=0.009)$ than in the FRE condition $(M=0.080, S E=0.004), p=0.009$. Additionally, the tpeak was significantly longer for $\operatorname{EXP}$ group $(M=0.10, S E=0.01)$ than for NOV group $(M=0.07, S E=0.01), F(1,18)=17.29, p=0.001$ (Figure 5). The group-bycondition interaction did not significantly affect the tpeak. 


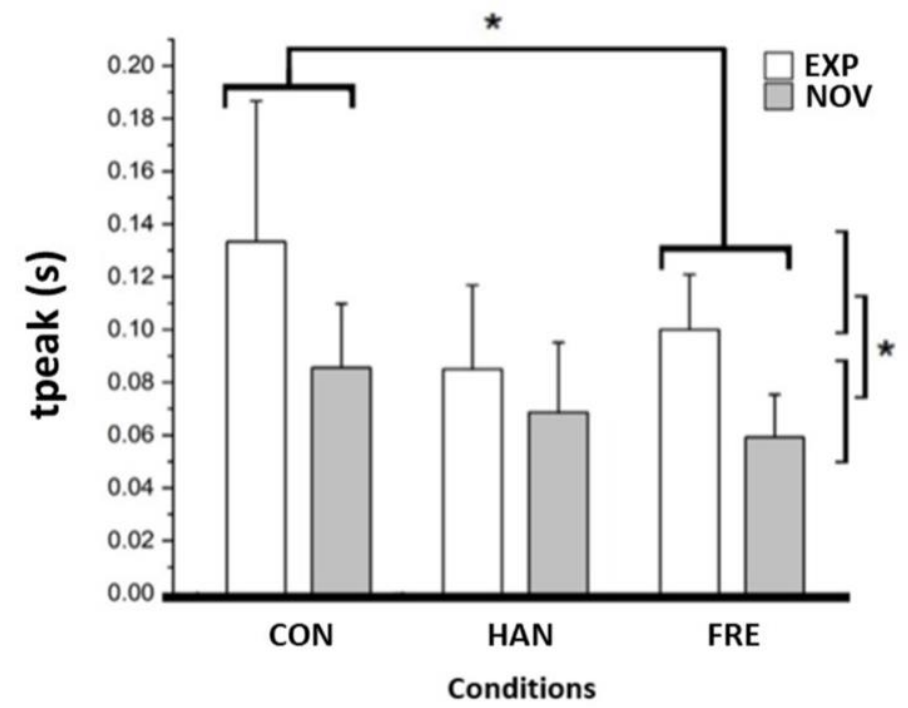

Figure 5. Mean (standard error) of tpeak (pixels) during control (CON), handheld (HAN), and hands-free (FRE) conditions of experienced (EXP) and novice (NOV) groups. ${ }^{*} p<0.05$.

\section{DISCUSSION}

This study successfully tested the adaptation and application of Klingner's method of fixation-aligned pupillary response averaging to the car driving context. In addition, based on this method, it determined effects of talking on a cell phone while driving, in both handheld and hands-free situations, and effects of driving experience on drivers' pupillary responses, as indicative of cognitive workload. Results showed that only NOV participants had their peak of pupillary response increased while talking on the cell phone, independently of holding it on their hands or using free-hand mode. Cognitive workload was modulated by experience and talking task.

One purpose of this study was to adapt and apply Klingner's method to the driving task. Overall, we argue that it was successful based on the following considerations: (i) as expected, we found that pupillary response average curves stood out from the noise measurement, as clearly shown in Figure 3. Participants' pupil dilated around the time of each fixation onset in all experimental conditions, indicating that this is a representative measure of cognitive workload. Given the constant light conditions in the laboratory, the considerably high number of fixations in each trial, and the baseline procedure applied to pupil data epoch, the method seemed adequate to associate pupil dilation information with cognitive activity involved in fixating distinct visual scene locations available while driving; (ii) although we rigorously followed Klinger's proposal, conducting temporal translation of epochs to guarantee fixation-aligned pupillary responses, the linear warping procedure was not used in the driving context.

This warping strategy basically adjusts the temporal duration across epochs, using a proportional relative time for each task subphase; for example, one of Klingner's task was map looking: warping procedure temporally aligned the subphases of looking at map symbols, looking at the legend, and looking back to the map symbols. In our case, this was 


\section{5 years of Brazilian Journal of Motor Behavior}

not necessary as the driving task was visually distributed to the whole road scene and distinct absolute durations of subphases did not represent a problem to confound pupillary response measurement; (iii) in the same vein as above, the driving task was visually and cognitively different from those used by Klingner; thus, the methodological adaptation used in the present study brought higher ecological validity to the pupil dilation analysis, considering a natural gaze behavior throughout the driving task.

As our method adaptation and application succeed, we were able to determine effects of talking on a cell phone while driving, in both handheld and hands-free situations, and effects of driving experience on drivers' pupillary responses, as indicative of cognitive workload. We hypothesized that talking on a cell phone while driving, in both handheld and hands-free situations, would significantly increase pupillary responses, mainly in the novice group. As we assessed pupillary responses in terms of its magnitude peak (peak) and the time to reach it (tpeak), we will discuss each dependent variable separately. Worth of note is the fact that the majority of studies use measures related to the magnitude of the pupil, such as diameter or dilation, without considering time of pupillary response variations (e.g. $\left.{ }^{13,14}\right)$.

In the present experiment, participants drove and talked on a cell phone, in handheld and hands-free situations (HAN and FRE conditions, respectively). These conversational dual-tasks (talking and driving) generated a higher cognitive workload only in the novice group, reflected in their peak of pupillary dilation, corroborating our hypothesis. This can be explained by the theory of multiple resources by Wickens, ${ }^{15}$ according to which two tasks requiring processing of the same dimension (i.e., visual perception and decoding) can interfere with each other, overloading the system. Conversation tends to evoke mental images to facilitate participants' line of reasoning and these moments of image evocation tend to disturb the visual interpretation of the scene during car driving, ${ }^{16}$ which can lead to the phenomenon of "looking and not seeing". 17 Particularly, cell phone conversations interfere with the detection of traffic scenes. ${ }^{18}$ Other sensory pathways may also be involved in cognitive workload; it has been shown that drivers' pupil diameter increased when performing an auditory task while driving. ${ }^{19}$ In the present study, both talking tasks using a cell phone required auditory processing, resulting in an increase of the peak of pupillary dilation. In short, present findings support previous studies indicating a linear relationship between pupil dilation and cognitive workload ${ }^{20}$ and the use of pupil dilation as an implicit metric of cognitive workload. ${ }^{21,22}$

Interestingly, HAN and FRE conditions did not differ in terms of pupil dilation peak of NOV participants, showing that cognitive engagement was more important than the mode of using the cell phone. This is in line with evidence from a study in a natural, realistic environment when participants drove a car equipped with a speakerphone and performed various cognitive tasks while driving, including talking to the passenger next to them; those findings did not suggest differences between live chat and cell phone chat tasks. ${ }^{19}$

Based on the present results, novice drivers "just talking" while driving do generate an increased cognitive workload, unlike the experienced ones, because their sensorimotor skills involved in car driving are not yet sufficiently accurate. When skills are automatized, less cortical processing is required from the individual, which makes more attentional resources available and allows improved performance. ${ }^{23}$ EXP group had the pupil dilation peak around $40 \%$ lower than NOV participants, which can be explained in part by the theory 


\section{5 years of Brazilian Journal of Motor Behavior}

of automation, which emphasizes that experienced drivers are capable of using divided attention and perform two tasks simultaneously. ${ }^{23}$

The temporal analysis of pupil reflected in time to reach its peak dilation (tpeak variable) brought additional aspects of cognitive workload debate. Using a cell phone seemed to reduce tpeak, although this difference reached significance only in FRE condition; additionally, EXP participants had their pupil dilation peak significantly later with respect to fixation onset (higher tpeak values). Although we did not include a dependent variable to specifically measure this aspect, it is observable from Figure 3 plots that the increase in pupil dilation above baseline level (zero value) started prior to fixation onset (time zero) in all conditions of both groups. Interestingly, Klingner ${ }^{7,8}$ also found pupil dilation increases prior to fixation onset when comparing a discovery of a target (first fixation to a specific target) to a revisit to it (later visits to the same target) during a visual search task; the author explained this finding by associating cognitive activity involved in the recall of previously identified target or saccade planning in order to reconfirm its location. In the present study, this anticipated pupil dilation with respect to fixation onset seemed connected to the relatively high cognitive workload of the driving task at high speeds $(80-120 \mathrm{~km} / \mathrm{h})$, which requires continuous visual inspection of a rapidly changing road conditions (and increased need of saccade planning) and timely coupled motor behavior (controlling the wheel, gears, checking mirrors and so on); particularly, tpeak significant differences found revealed that higher workload anticipated tpeak values due to the task demands (condition effect) and to participants' driving experience (group effect), suggesting that these aspects are dependent on the dynamics of memory and experience..$^{7,8,13}$ In summary, it seems reasonable to speculate that the planning of saccadic movements, the uncertainties of eye control, and the relatively greater effort for beginners to carry out the task of driving (particularly when they were talking on the cell phone) were more costly from the cognitive point of view, generating earlier onset of pupil dilation and a peak of greater magnitude, with less time to reach it among novices.

\section{CONCLUSION}

Overall, we conclude that present results supported our hypotheses. First, adaptation and application of Klingner's method of fixation-aligned pupillary response averaging to the car driving context was successful. Second, pupil dilation was a robust indicative of cognitive workload while driving a car; talking on a cell phone while driving, in both handheld and hands-free situations, significantly caused increased pupillary dilation, mainly in the novice group. Possible applications of our findings include future automotive technologies to online automatic detection of risky cognitive workload while driving from pupil dilation and eye movements simultaneous data (from video sources, possibly combined with other biological signals such as heartbeat), calibrated to drivers' skill level, in order to prevent accidents. 


\section{REFERENCES}

1. Wel PVD, Van Steenbergen $H$. Pupil dilation as an index of effort in cognitive control tasks: a review. PBR. 2018; 25: 2005-2015. doi: 10.3758/s13423-018-1432-y 301

2. Yeung MK, Lee TL, Han YMY, Chan AS. Prefrontal activation and pupil dilation during $n-$ back task performance: a combined fnirs and pupillometry study. Neuropsychologia. 2021; 159: 107954. doi: 10.1016/j.neuropsychologia.2021.107954

3. Caird JK, et al. A meta-analysis of the effects of cell phones on driver performance. Accid Anal Prev. 2008; 40(4): 1282-1293. doi: 10.1016/j.aap.2008.01.009

4. Chapman PR, Underwood G. Visual search of driving situations: danger and experience. Perception. 1998; 27: 951-964. doi: 10.1068/p270951

5. Vollrath $\mathrm{M}$, Clifford $\mathrm{C}$, Huemer AK. Even experienced phone users drive worse while texting: a driving simulator study. Transp Res F: Traffic Psychol Behav. 2021; 78: 218-225. doi: 10.1016/j.trf.2021.02.007

6. Bitkina OV, Park J, Kim HK. The ability of eye-tracking metrics to classify and predict the perceived driving workload. Int J Ind Ergon. 2021; 86: 103193. doi: 10.1016/j.ergon.2021.103193

7. Klingner J. Measuring cognitive load during visual tasks by combining pupillometry and eye tracking. Stanford: Stanford University; $2010.130 \mathrm{p}$.

8. Klingner J. Fixation-aligned Pupillary Response Averaging. ETRA. 2010 b.; 22: 275-282. doi: $10.1145 / 1743666.1743732318$

9. Lehtonen $\mathrm{E}$, Lappi $\mathrm{O}$, Koirikivi I, Summala $\mathrm{H}$. Effect of driving experience on anticipatory look-ahead fixations in real curve driving. Accid Anal Prev. 2014; 70: 195-208. doi: 10.1016/j.aap.2014.04.002

10. Summala H, Nieminen T, Punto M. Maintaining lane position with peripheral vision during in-vehicle tasks. Hum Factors: The Journal of the Human Factors and Ergonomics Society.1996; 38(3): 442-451. doi: 10.1518/001872096778701944

11. Wong PKH, Bickford RG. Brain stem auditory evoked potentials: the use of noise estimate. Electroencephalogr Clin Neurophysiol. 1980; 50:25-34.

12. Schimmel H. Accuracy of estimated mean components in average response studies. Science.1967; 3784(157):92-94. doi: 10.1126/science.157.3784.92

13. Kim JH, Yang $X$. Measuring driver's perceived workload using fractal dimension of pupil dilation. Proceedings of the 2020 HFES 64th International Annual Meeting. 2020; 64: 1620-1624. doi: 10.1177/1071181320641392

14. Marinescu AC, et al. Physiological parameter response to variation of mental workload.2018; 60(1): 31-56. doi: 10.1177/0018720817733101

15. Wickens CD. Multiple resources and performance prediction. Theor Issues Ergon Sci. 2002; 3(2): 159-177. doi: 10.1080/14639220210123806

16. Barsalou LW. Perceptual symbol systems. Behav Brain Sci.1999; 22: 577-609. 
17. Briggs GF, Hole GJ, Land MF. Imagery-inducing distraction leads to cognitive tunnelling and deteriorated driving performance. Transp Res F: Traffic Psychol Behav. 2016; 38:106117. doi: 10.1016/j.trf.2016.01.007

18. Mccarley JS, et al. Conversation disrupts change detection in complex traffic scenes. Hum Factors. 2004; 46(3): 423-436.

19. Nunes $L$, Recarte MA. Cognitive demands of hands-free-phone conversation while driving. Transp Res F: Traffic Psychol Behav. 2002; 5(2):133-144. doi: 10.1016/s13698478(02)00012-8

20. Kahneman D, Beatty J. Pupil diameter and load on memory. Science.1966; 154: 15831585.

21. Halverson T, Estepp J, Christensen J, Monnin J. Classifying workload with eye movements in a complex task. Proc Hum Factors Ergon Soc Annu Meet. 2012; 6 (1): 168172. doi: $10.1177 / 1071181312561012$

22. Edirisinghe VA. Estimating mental workload of university students using eye parameters. Trondheim: Norwegian University of Science and Technology; 2017. 88 p.

23. Sternberg RJ. Psicologia cognitiva. Porto Alegre, RS: Artmed; 2000. 494 p.

\section{ACKNOWLEDGMENTS}

We thank Tiago Chinez Simões for translating the article into English.

Citation: Carizio BG, Silva GA, Paschoalino GP, Angelo JC, Gotardi GC, Zago PFP, Rodrigues ST. (2021). Pupil dilation as indicative of cognitive workload while driving a car: Effects of cell phone use and driver experience in young adults. Brazilian Journal of Motor Behavior, 15(5):391-402.

Editors: Dr Fabio Augusto Barbieri - São Paulo State University (UNESP), Bauru, SP, Brazil; Dr José Angelo Barela São Paulo State University (UNESP), Rio Claro, SP, Brazil; Dr Natalia Madalena Rinaldi - Federal University of Espírito Santo (UFES), Vitória, ES, Brazil.

Copyright:@ 2021 Carizio, Silva, Paschoalino, Angelo, Gotardi, Zago and Rodrigues and BJMB. This is an openaccess article distributed under the terms of the Creative Commons Attribution-Non Commercial-No Derivatives 4.0 International License which permits unrestricted use, distribution, and reproduction in any medium, provided the original author and source are credited.

Funding: This work was supported by the National Council for Scientific and Technological Development - CNPq, Brazil (N¹33543/2014-5).

Competing interests: The authors have declared that no competing interests exist.

DOI: https://doi.org/10.20338/bjmb.v15i5.269 